\title{
Counting the Quanta of Sound
}

\section{Two teams demonstrate that they can count the number of quantized vibrations, or phonons, in cold mechanical oscillators by measuring the energy in the vibrations.}

\section{by Laure Mercier de Lépinay* and Mika A. Sillanpää†}

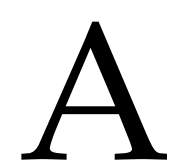

$t$ the origin of every musical note is a mechanical oscillator that resonates at a specific frequency. But what the ear cannot distinguish is that the energy of these vibrations is discretized into an integer number of quanta of motion, or phonons. Most vibrating objects contain an uncountable number of phonons, but researchers have, for some time now, been able to prepare massive mechanical oscillators in their quantum ground state, where the average phonon number is smaller than one. This hard-won accomplishment not only involved getting rid of all thermal excitations in the oscillator through intense cooling, but it also required inventing a system of motion detection with a sensitivity at the quantum level [1]. An emerging technique consists of coupling the oscillator motion to another quantum object: a superconducting qubit, which can serve a role in the detection as well as the manipulation of states of motion [2-4]. Using such a "qubit sound

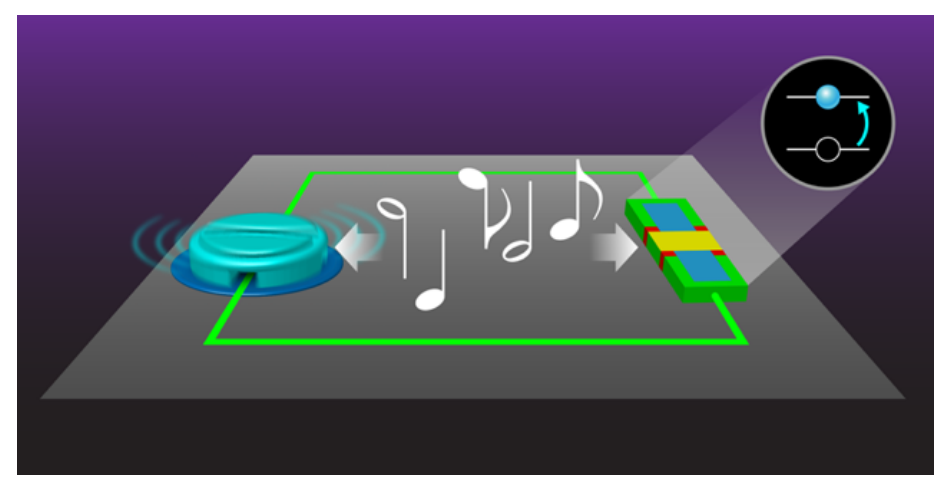

Figure 1: Measuring the number of phonons, or "sound quanta," in a mechanical oscillator (left) can be done by connecting it to a superconducting qubit (right), as demonstrated in two new experiments $[5,6]$. In one case, the oscillator is a vibrating drumhead, as depicted here, while in the other, the oscillator is an acoustic resonator. Both experiments use distinct quantum transition energies in the qubit to address individual phonon states in the oscillator. (APS/Alan Stonebraker)

*Department of Applied Physics, Aalto University, Aalto, Finland $\dagger$ Department of Applied Physics, Aalto University, Aalto, Finland system," two separate teams have managed to measure the number of phonons directly in a macroscopic mechanical oscillator. In one case, the oscillator is a membrane whose center of mass vibrates like a drumhead [5], while in the other case, the oscillator is a type of sound-wave cavity called an acoustic wave resonator [6]. By demonstrating unprecedented control over states of motion, these results may open the door to the use of oscillators as gravity sensors and quantum memory devices.

The motivation behind the two groups' efforts is to measure and control so-called Fock states that are characterized by a definite phonon number. The set of Fock states includes the zero-phonon state, the one-phonon state, the two-phonon state, and so on. Some previous experimenters operated mechanical oscillators near their ground states, in which case one can infer that the systems were predominantly in the zero-phonon Fock state. But in most other experiments, researchers have not measured Fock states directly. Instead, they have estimated an average phonon number (or average energy) by observing the oscillator's position, momentum, or both.

Even though their aims are similar, the two groups have very different strategies and methods. Jeremie Viennot and colleagues at the University of Colorado, Boulder, studied the motion of an aluminum membrane that was a few micrometers across [5] (Fig. 1). This vibrating "drumhead" is categorized as a center-of-mass oscillator, meaning that a macroscopic part of the object is elastically displaced [7]. This displacement happens at relatively low frequencies in the megahertz range, immensely different from the gigahertz transition frequencies of superconducting qubits. The Boulder team therefore faced a major challenge in engineering a strong off-resonant coupling between their oscillator and a superconducting qubit. The type of superconducting qubit that they used was a charge qubit, whose states are identified by the presence of excess Cooper pairs in a small island [8]. Charge qubits were considered prime candidates for coupling to mechanical oscillators [2, 3], but they are infamously sensitive to movements of charges in the surroundings. The Boulder team managed to operate their qubit in a way that was insensitive to environmental charges, while still having a nonresonant interaction with charges that move in response to the mechanical oscillator's vibrations.

As a result of this off-resonant interaction, the frequency 
of the qubit should be shifted in proportion to the number of phonons in the oscillator. The researchers managed to make the one-phonon-induced shift large enough, and the qubit resonance narrow enough, that only seven phonons suffice to shift the qubit resonance by its linewidth. When the team measured the qubit spectrum with standard techniques, they could discern the probability that the system was in a particular Fock state. Notably, this measurement technique-which was invented to measure Fock state distributions for a microwave resonator [9] and is implemented here for the first time in a mechanical setting-does not destroy the fragile state of the mechanical oscillator.

The Boulder team went beyond just identifying Fock states; they also used the qubit to pump phonons into and out of the oscillator. For example, if they drove the qubit at a frequency larger than its resonance frequency by exactly the mechanical frequency, then this excess of energy was converted into excitations of the mechanical oscillator. These excitations could be targeted for specific number states, allowing the authors to nearly empty the Fock state distribution at particular phonon numbers by shoving the initial populations of these states toward higher phonon numbers. Conversely, driving the qubit at a frequency lower than its resonance frequency pumped phonons out of the mechanical oscillator. Putting this into practice, the researchers could shift the weight of the Fock state distribution toward lower phonon numbers, creating a high zero-phonon ground-state population in a manner distinct from any earlier work.

Interestingly, Yiwen Chu and colleagues at Yale University were able to do similar manipulations but in a very different type of system [6]. The oscillator studied by the Yale group was a half-millimeter-thick sapphire chip, which supports a propagating sound wave but does not have macroscopic moving parts. Such acoustic resonators vibrate at much higher frequencies in the microwave range, allowing them to be addressed resonantly with qubits. These higher frequencies also mean that cryogenic cooling suffices to eliminate all thermal excitations and reach the motional quantum ground state.

In their resonantly coupled system, the Yale team managed to swap excitations coherently between the qubit and the mechanical oscillator. In these kinds of experiments, if the oscillator is initially in the quantum ground state, the swapping of quanta takes place at the so-called vacuum Rabi frequency [10]. However, in the Yale team's experiments, the Rabi frequency attained an individual discrete value for each Fock state higher than zero. By measuring these Rabi oscillations of the qubit state, the researchers could therefore extract the phonon Fock state occupancies from the frequency components. When the oscillator was in its ground state, the team could prepare the qubit in its excited state and perform the exchange, causing the phonon number to increase by one. By iterating this "phonon stepping" process multiple times, they could prepare the mechanical oscillator in a high-phonon-number Fock state.

The experiments both show an unprecedentedly high degree of control over states of motion. And each strategy has its advantages. The Boulder team's method measures phonons nondestructively, whereas the Yale group's resonant readout irreversibly destroys the mechanical state. However, the Yale group's preparation scheme can yield high-purity Fock states, which can be good for quantum information. Taken together, the two categories of oscillators span a broad range of mechanical frequencies, which makes them complementary for applications. Manipulating massive quantized center-of-mass motion would enable researchers to probe the unexplored grey area between the microscopic quantum world and our familiar observable world. Thanks to their extreme delicacy, these states of motion might also prove sensitive to gravity at small scales. And finally, because of their high coherence and easy integration into other physical systems, quantum states of mechanical oscillators are considered excellent candidates for memory storage in quantum information technologies and for converting between electronic signals and optical signals.

This research is published in Physical Review Letters and on the arXiv.

\section{REFERENCES}

[1] A. D. O'Connell et al., "Quantum ground state and singlephonon control of a mechanical resonator," Nature 464, 697 (2010).

[2] M. D. LaHaye, J. Suh, P. M. Echternach, K. C. Schwab, and M. L. Roukes, "Nanomechanical measurements of a superconducting qubit," Nature 459, 960 (2009).

[3] J.-M. Pirkkalainen, S.U. Cho, F. Massel, J. Tuorila, T.T. Heikkilä, P.J. Hakonen, and M.A. Sillanpää, "Cavity optomechanics mediated by a quantum two-level system," Nat. Commun. 6, 6981 (2015).

[4] A. P. Reed et al., "Faithful conversion of propagating quantum information to mechanical motion," Nat. Phys. 13, 1163 (2017).

[5] J. J. Viennot, X. Ma, and K. W. Lehnert, "Phonon-numbersensitive electromechanics," Phys. Rev. Lett. 121, 183601 (2018).

[6] Y. Chu et al., "Climbing the phonon Fock state ladder," arXiv:1804.07426.

[7] J. D. Teufel, T. Donner, Dale Li, J. W. Harlow, M. S. Allman, K. Cicak, A. J. Sirois, J. D. Whittaker, K. W. Lehnert, and R. W. Simmonds, "Sideband cooling of micromechanical motion to the quantum ground state," Nature 475, 359 (2011).

[8] Y. Nakamura, Yu. A. Pashkin, and J. S. Tsai, "Coherent control of macroscopic quantum states in a single-Cooper-pair box," Nature 398, 786 (1999).

[9] D. I. Schuster et al., "Resolving photon number states in a superconducting circuit," Nature 445, 515 (2007).

[10] M. Brune, F. Schmidt-Kaler, A. Maali, J. Dreyer, E. Hagley, J. M. Raimond, and S. Haroche, "Quantum Rabi oscillation: A direct test of field quantization in a cavity," Phys. Rev. Lett. 76, 1800 (1996). 
Phys̄î̄CS

10.1103/Physics.11.109 